\section{Wising up to NPYs}

\section{By Lev Osherovich, Senior Writer}

In the mid-1990s, neuropeptide $\mathrm{Y}$ was thought to be a promising obesity target based on its activity in the CNS as a potent feeding stimulator. However, the approach didn't succeed and only one company is still working in that space. But in a turn of events, two new studies now suggest that the peptide hormone could actually be a target in atherosclerosis and coronary artery disease.

One study, published in Public Library of Science Genetics by a team at Duke University School of Medicine, links mutations near the gene encoding neuropeptide Y (NPY) to higher risk of early onset CAD. ${ }^{1}$

A second study, published in the Journal of Neuroscience, examined the synthesis and secretion of an NPY variant associated with atherosclerosis in Scandinavian families. ${ }^{2}$

NPY and its receptors traditionally have been studied in the CNS, where they regulate appetite, emotion and libido. However, previous rat studies did hint that NPY signaling could affect recovery from arterial injury, which can trigger CAD. ${ }^{3}$

The Duke study shores up NPY's cardiovascular function with human genomewide association data and experiments in mice linking the peptide and one of its receptors to the development of atherosclerosis.

"The strength of our study is that we combine a lot of methods to clinch the case for neuropeptide $\mathrm{Y}$ in coronary artery disease," said Svati Shah, assistant professor of medicine at Duke and the lead author of the study.

\section{Risky linkage}

$N P Y$ is the latest of several CAD risk genes identified by Shah's team using DNA from a panel of families with a high incidence of earlyonset CAD. In an earlier study called GENECARD, the Duke team and partners at GlaxoSmithKline plc identified several chromosomal regions with markers that correlated with CAD onset at an early age. ${ }^{4}$ GSK was not involved with the new study, according to a spokesperson.

The GENECARD study was published in 2004, and the Duke team has since whittled the relatively broad disease-linked chromosomal regions down to candidate genes, thanks to improved marker density and statistical methods.

Shah told SciBX that the NPY locus was not the strongest of the hereditary CAD signals in the study. This is likely because only a small fraction of subjects in the study carried NPY-linked mutations compared with more common mutations in other CAD risk genes.
However, when Shah's team focused on individuals with the earliest mean age of CAD onset, the NPY locus emerged as the most significant risk factor.

NPY is expressed as a prohormone that undergoes proteolytic processing to produce the 36-amino-acid peptide. The CAD-associated mutations were in the promoter region of the NPY gene, and as a result the team postulated that the mutations could affect the gene's expression. Indeed, in 220 randomly chosen study subjects drawn from disease and control groups, carriers of the risk allele had higher levels of plasma NPY than noncarriers $(46.3 \mathrm{pmol} / \mathrm{L}$ vs. $41 \mathrm{pmol} / \mathrm{L}$, $p=0.04)$.

\section{Secretes revealed}

The Duke study dovetails with the report in the Journal of Neuroscience that describes the mechanism of a rare NPY mutation found in certain Scandinavian families. That allele causes high levels of serum cholesterol and atherosclerosis, as well as increased risk of obesity and diabetes. ${ }^{5}$

Matthew Whim, assistant professor of biology at Pennsylvania State University and team leader for the study, told SciBX that the Scandinavian mutation occurs in the signal sequence, a region at the beginning of the prohormone that guides the NPY precursor into the secretory pathway. The signal sequence is eventually removed by proteolytic processing, leading to secretion of NPY by activated neurons.

His team found that cultured mouse cells expressing the mutant Npy precursor secrete the hormone more vigorously in response to electrical stimulation than wild-type controls. Like the mutations found by the Duke group, the Scandinavian mutation leads to an increase in overall NPY production, albeit by a different mechanism.

The finding that "a common polymorphism in the NPY gene leads to an increase of release" of the hormone is a surprise, said Whim. Typically, mutations in the signal sequence cause decreases in hormone processing and secretion.

In light of the two studies, Whim said it would be worthwhile to test the effect of the NPY promoter mutations identified by Shah in a cell culture model of NPY secretion such as the one used by Whim's team.

\section{Brain or body?}

Together, the studies make the case that excess NPY activity plays a role in atherosclerosis and CAD. The challenge now is to figure out where NPY acts and how to lower levels of NPY or antagonize the appropriate NPY receptors.

Sheng $\mathrm{Bi}$, assistant professor of psychiatry at Johns Hopkins School of Medicine, noted that most previous work has focused on NPY's role in the hypothalamic regulation of appetite. Indeed, he published a study of NPY-induced food intake in the dorsomedial hypothalamus in the Journal of Neuroscience this month. ${ }^{6}$

"NPY is orexigenic, promoting food intake and eventually obesity," 


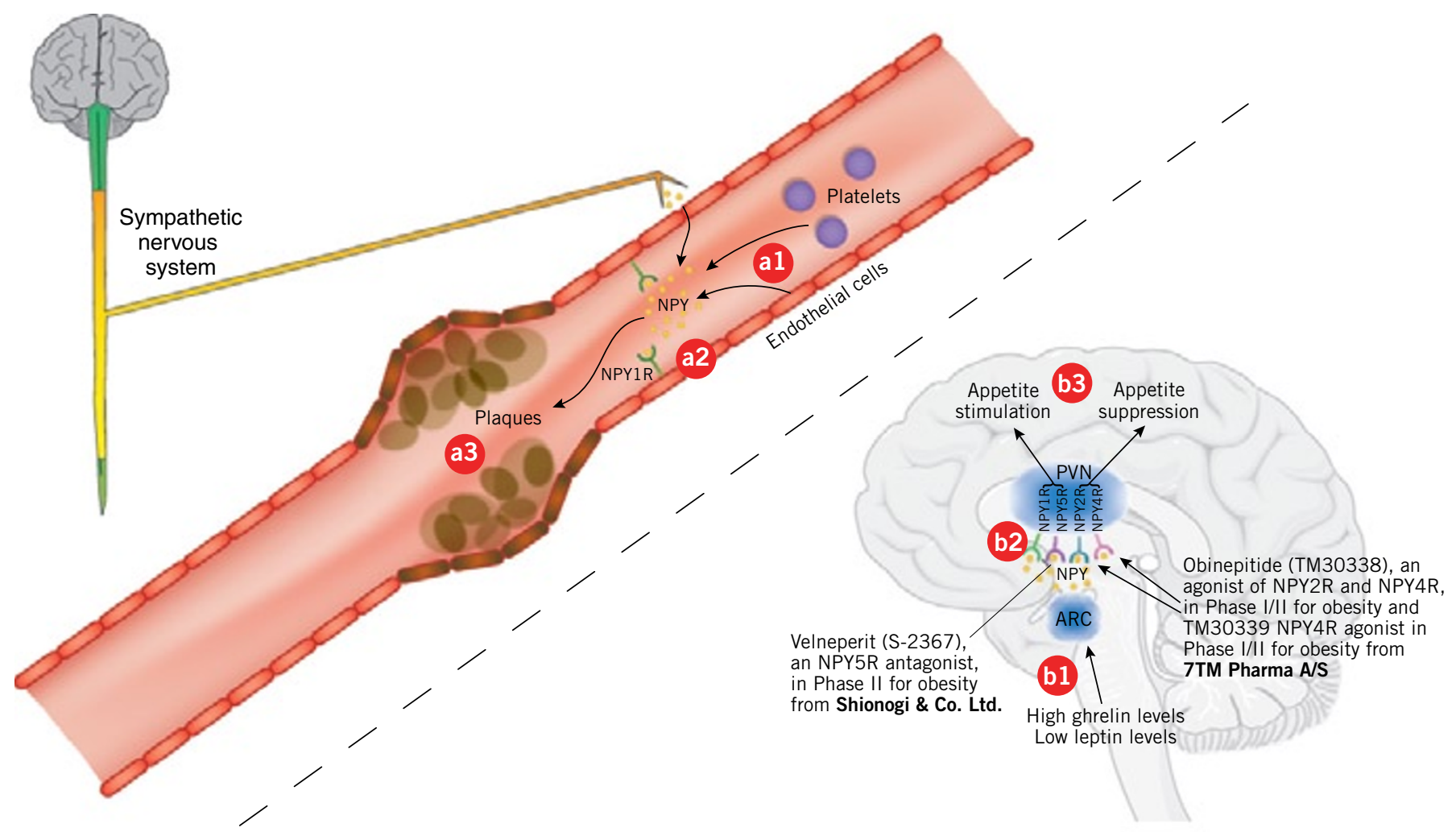

Figure 1. Neuropeptide $Y$ in atherosclerosis and obesity. Shah et al. have found that, independent of neuropeptide $Y$ (NPY)'s role in the brain, blocking the peptide hormone in the vasculature could be useful for treating atherosclerosis. In humans, early-onset coronary artery disease (CAD) is correlated with mutations in the NPY gene that lead to greater NPY production. In mice, antagonists of Npy receptor Y1 (Npy1r) prevent atherosclerosis.

These finding suggest that NPY, which is secreted by platelets, endothelial cells and neurons of the sympathetic nervous system [a1], could activate NPY1R in an unknown cell type within the artery [a2], leading to local inflammation and plaque deposition [a3].

In the brain, NPY is associated with appetite control. Neurons in the arcuate nucleus (ARC) of the hypothalamus produce NPY when levels of the gut-derived hormone ghrelin are high and levels of the adipocyte-derived hormone leptin are low [b1]. NPY activates a range of NPY receptors (NPY1R, NPY2R, NPY4R and NPY5R) in the paraventricular nucleus (PVN) and other regions of the hypothalamus [b2], with opposing effects on appetite. NPY1R and NPY5R are tied to appetite stimulation, whereas NPY2R and NPY4R are tied to appetite suppression [b3].

said $\mathrm{Bi}$. Thus, the big question is whether the effects of excess NPY on CAD and atherosclerosis occur in the CNS or in the sympathetic nervous system that innervates the vasculature (see Figure 1, "Neuropeptide $Y$ in atherosclerosis and obesity").

One scenario is that higher NPY levels could prompt food cravings that lead to poor diet and greater CAD risk. Indeed, risk allele carriers in Shah's study also had higher triglyceride levels and a higher incidence of dyslipidemia and diabetes than noncarrier controls. However, Shah noted that in her human sample set, there was no correlation between the high-risk NPY allele and obesity.

Instead, Shah favors a role for NPY in the vasculature. She cited mouse experiments in the PLoS Genetics paper showing that local inhibition of NPY receptor Y1 (NPY1R) with a small molecule lowered the severity of injury-induced smooth muscle proliferation in the coronary artery compared with that seen in mock-treated controls.

These mouse studies "create a strong link for the pathway of NPY protein and the NPY1 receptor" acting locally in arterial disease, said Shah.

To resolve where NPY acts, Bi recommended further experiments in rodents to examine the effect of blocking NPY or NPY receptors in various parts of the hypothalamus or cardiovascular system. Meanwhile, Whim suggested measuring local NPY levels near atherosclerotic plaques.

\section{Applications}

NPY therapeutics thus far have targeted a family of G protein-coupled receptors that sense the hormone in the $\mathrm{CNS}$ and have both hungerpromoting and inhibiting effects depending on the specific receptor subtype. However, NPY receptor antagonist therapies for obesity have failed in the clinic.

Merck \& Co. Inc. terminated its MK-0557 compound, an antagonist of NPY5R, after disappointing results in Phase II obesity tri- 
als, and the company is no longer developing the compound, according to a spokesperson. MDRNA Inc. suspended development of PYY3-36 following the failure of the nasal spray formulation of an NPY receptor antagonist in Phase II obesity trials. Likewise, $\mathbf{H}$. Lundbeck A/S discontinued a Phase I trial of its NPY receptor antagonist for mood disorders in January 2008.

Shionogi \& Co. Ltd. is continuing its effort, as its Velneperit (S2367) NPY5R antagonist is in Phase II trials for obesity.

But if obesity has proved difficult, the new papers could broaden the therapeutic opportunities for compounds from 7TM Pharma A/S, which has explored NPY2R and NPY4R modulators for metabolic and cardiovascular indications.

The PLoS Genetics study "highlights the rich pharmacology displayed by NPY and the NPY receptor family," said Christian Elling, VP of development at 7TM. The Shah study establishes "the link between NPY and coronary artery disease conceivably through the NPY receptor Y1."

The company's obinepitide, an agonist of NPY2R and NPY4R, is in Phase II trials to treat obesity and metabolic disorders. Elling told SciBX that $\mathrm{Y} 2$ and $\mathrm{Y} 4$ receptors may have opposite effects on appetite compared with the $\mathrm{Y} 1$ and $\mathrm{Y} 5$ receptors targeted by other companies, and thus they need to be agonized rather than antagonized to treat obesity.

Elling added that 7TM is already investigating potential cardiovascular effects of its NPY receptor agonists.

"Y2 agonists may have beneficial effects on angiogenesis and arteriogenesis, which 7TM is pursuing via a collaboration with Procter \& Gamble Co.," said Elling. Under that 2005 deal, the biotech and $P \& G$ are jointly developing compounds for cardiovascular indications.

Although risk of CAD can be decreased with lifestyle changes and cholesterol-lowering statins, advanced CAD is treated primarily with angioplasty or drug-eluting stents. Shah therefore thinks that stents that elute NPY receptor inhibitors could be a new option to prevent thrombosis and restenosis, a common side effect of stenting in which smooth muscle cells proliferate and clog the devices.

"If you could prevent the atherosclerotic process as well as smooth muscle proliferation, you could potentially prevent recurrence of atherosclerosis," she said.

Neither Shah nor Whim have patented their findings.

Osherovich, L. SciBX 2(4); doi:10.1038/scibx.2009.123

Published online Jan. 29, 2009

\section{REFERENCES}

1. Shah, S.H. et al. PLoS Genet. published online Jan. 2, 2009; doi:10.1371/journal.pgen.1000318

Contact: Svati H. Shah, Duke University School of Medicine, Durham, N.C. e-mail: svati.shah@duke.edu

2. Mitchell, G.C. et al. J. Neurosci. published online Dec. 31, 2009; doi:10.1523/JNEUROSCI.0343-08.2008

Contact: Matthew D. Whim, Pennsylvania State University, State College, Pa. e-mail: mdw13@psu.edu

3. Li, L. et al. Arterioscler. Thromb. Vasc. Biol. 25, 2075-2080 (2005)

4. Hauser, E.R. et al. Am. J. Hum. Genet. 75, 436-447 (2004)

5. Karvonen, M.K. et al. Nat. Med. 4, 1434-1437 (1998)

6. Yang, L. et al. J. Neurosci. 29, 179-190 (2009)

COMPANIES AND INSTITUTIONS MENTIONED

7TM Pharma A/S, Horsholm, Denmark Duke University School of Medicine, Durham, N.C. GlaxoSmithKline plc (LSE:GSK; NYSE:GSK), London, U.K. H. Lundbeck A/S (CSE:LUN), Copenhagen, Denmark Johns Hopkins School of Medicine, Baltimore, Md. MDRNA Inc. (NASDAQ:MRNA), Bothell, Wash. Merck \& Co. Inc. (NYSE:MRK), Whitehouse Station, N.J. Pennsylvania State University, College Station, $\mathrm{Pa}$. Procter \& Gamble Co. (NYSE:PG), Cincinnati, Ohio Shionogi \& Co. Ltd., Osaka, Japan 